\title{
A new concept to improve the lithium plating detection sensitivity in lithium-ion batteries
}

\author{
Upender Rao Koleti *, Cheng Zhang , Truong Quang Dinh , James Marco , \\ Tazdin Amietszajew , Romeo Malik
}

WMG, The University of Warwick, Coventry CV4 7AL, United Kingdom

\begin{abstract}
Lithium plating significantly reduces the lifetime of lithium-ion batteries and may even pose a safety risk in the form of an internal short circuit, leading to catastrophic cell failure. Low temperatures, high charge currents and battery age are known to be contributing factors to increased lithium plating. To reduce or avoid battery ageing induced by lithium plating, a method for lithium plating detection is essential to help understand the favourable conditions under which battery charging is optimised. In this study, we present a concept to design an experiment-based approach to improve lithium plating detection sensitivity using the non-destructive voltage relaxation analysis method. Commercial NCA/graphite cells are employed for this study. Here, the reversible part of the plated lithium providing a unique cell voltage relaxation profile is used as a pseudo-measure to detect the onset of plating. This profile is observed while the cell is at rest as well as under a low C-rate discharge regime immediately after charging. It is found that the CV (Constant Voltage) phase cut-off current value significantly influences plating detection. To address this issue, a procedure to determine the optimal cut-off current in the CV phase of charging is introduced to improve the detection sensitivity. With the proposed method, plating detection chances are improved which will help in understanding the favourable conditions and developing plating control strategies. Furthermore, a correlation between lithium plating and Electrochemical Impedance Spectroscopy (EIS) measurements are utilized to demonstrate the influence of previous ageing conditions on lithium plating.
\end{abstract}

Keywords: Lithium plating, Battery ageing, Lithium stripping, Reversibility, Capacity fade

\section{Introduction}

Lithium-ion batteries are used in portable electronic devices and electric vehicles (EVs) because of their high specific energy and long cycle life. However, the battery life is still a bottleneck on the penetration of EVs in the automotive sector. Lithium batteries lose performance in the form of capacity and power fade with usage and storage time[1]. Among several ageing mechanisms, such as Solid Electrolyte Interface (SEI) layer growth and mechanical stresses, lithium metal deposition on the graphite negative electrode that occurs during charging is of great significance because it was associated with an accelerated degradation [2, 3]. Low temperatures $\left(<25^{\circ} \mathrm{C}\right)$, high charge currents $(>1 \mathrm{C})$ and aged conditions increase the negative electrode potential drop because of reduced kinetics [3-5]. When the electrode potential drops below the $\mathrm{Li}+$ reference potential, lithium metal deposition starts on the electrode in addition to the lithium intercalation into the graphite. Part of the plated lithium is reversible which returns to the electrode, while the rest may react with the electrolyte (adding to SEI growth) or becomes electrically disconnected from the electrode creating a pool of inactive metallic lithium[6]. Overall, capacity loss and impedance rise can be observed as a consequence of lithium plating. Lithium plating may also create safety issues if dendrites are formed out of the isolated lithium metal. In the worst case, such dendrites may cause an internal short circuit as the dendrites pierce the separator $[2,7]$.

\footnotetext{
* Manuscript received February 15, 2018; revised April 15, 2019.

Upender Rao Koleti. Tel.:+44-7471114926; E-mail address: u.koleti@warwick.ac.uk.

doi: $10.12720 /$ sgce.8.5.505-516
} 
For all these reasons, it is essential to avoid lithium plating. To control lithium plating it is necessary to understand the conditions favourable for it to occur. Thus, quantification of lithium plating is a prerequisite. Both parts of the plated lithium need to be quantified or detected, because reversible part of the plated lithium may cause capacity loss indirectly by the mechanical stresses it creates[8] while the irreversible part leads to a direct loss of lithium[9, 10].

Tomography, Voltage relaxation profiles(VRFs) and Differential curves(DV) are employed to estimate the reversible part of the plated lithium. These non-destructive methods are based on the observable features that occur in the cell immediately after the plating. Post-charge, graphite electrode potential starts to recover and as soon as the graphite potential exceeds the $\mathrm{Li} / \mathrm{Li}+$ potential, intercalation of the plated lithium into graphite will start [11]. The rise in the electrode lithiation level indicates the amount of lithium returned and is measured by neutron diffraction based Tomography technique[11]. However, this method cannot be applied in a practical application because of the machinery it uses. Alternatively, the influence of reversible plating on the cell voltage in the post-charge conditions can also be used to detect and quantify the reversible part of plating in two different ways: the one by low c-rate discharge and the second, by the rest. In the first method, a high voltage plateau due to reversible plating during low c-rate discharge was observed and used to detect the plating $[5,12]$. Reversible part of the plated lithium while contributing to the load current produces a voltage plateau compared to the usual delithiation of the graphite electrode. This method is applied to an LFP cell where negative electrode phase changes are observed by tracking the cell voltage since the positive electrode is having a nearly constant voltage profile. Yet, use of such a method may not guarantee the plating detection on the NMC and NCA cells which exhibit a varying positive electrode voltage profile. In the second approach, plating produces a distinct cell voltage relaxation profile (VRF) in the post-charge rest period[12, 13]. As long as this reversing of the lithium plating occurs, the potential of the negative electrode remains constant. The usual voltage relaxation behaviour of the positive electrode and the unusual behaviour of the negative electrode potential shall produce a unique feature on the cell voltage. The differential on the cell voltage allows us to identify the time to complete reversing reactions. Christian et al. has shown that the VRFs can be used to quantify the lithium plating [13] by comparing the VRFs with tomography results.

To detect or estimate the irreversible plating, there are various methods such as post-mortem analysis, Coulombic efficiency and capacity fade. Post-mortem analysis through Scanning Electron Microscope(SEM)[14] and Inductively Coupled Plasma Optical Emission Spectrometry (ICP-OES)[15] were used to study the non-reversible part of the plating that remains in the cell after it is opened. However, they are not suitable for finding the plating effects cycle by cycle in order to avoid plating conditions in real-time operation. Burns et al. have proposed a non-destructive method based on high precision Coulomb counting to detect the changes to the Coulombic efficiency during cycling resulting from small amounts of lithium plating [16, 17]. Coulombic efficiency is the ratio of charge output compared to the charge input. Loss of cyclable lithium resulting from lithium plating reduces the Coulombic efficiency, which is used as a measure to estimate lithium plating. High accuracy in current measurement (in the order of $0.01 \%$ ) to calculate the Couloumbic efficiency makes this method difficult to use in real time application [18]. Alternatively, the capacity fade exhibited by the cell can be used to estimate the irreversible lithium plating [5, 19]. This approach requires knowledge of the capacity drop trends exhibited by the other ageing mechanism such as SEI growth and loss of active material(LAM) to attribute the additional change to lithium plating.

Since none of these reported methods can detect the amounts of both the reversible and irreversible plated lithium, a plating study needs to use at least one reversible and one irreversible plating detection method to yield a complete understanding [5, 19]. Petzl et al. have used DV curves for the reversible plating estimation and capacity fade method to quantify the irreversible lithium plating[19]. When reversible plating is detected, the irreversible part is estimated to fully understand the total amount of lithium plating. This indicates that the reversible plating detection is crucial for the total amount of lithium plating estimation. Improving the reversible plating detection method's sensitivity to plating levels helps to sense plating at the inception. 
Previously, plating studies were performed on the NMC and LFP cells at sub-zero temperatures with a $\mathrm{CV}$ phase cut-off current close to zero $(\mathrm{C} / 40)[11,12]$. Under such low current cut-off values, a portion of the plated lithium may reverse in the $\mathrm{CV}$ phase itself. The negative electrode voltage reaches a minimum at the end of $\mathrm{CC}$ phase and starts recovering in the $\mathrm{CV}$ phase $[3,20]$. If the cut-off current in the $\mathrm{CV}$ phase is sufficiently small, the negative electrode potential will become positive which initiates the reversing. Still, the detection was possible because of the large lithium plating levels and slow reversing reactions due to the low temperature. While testing at higher temperatures, the lithium plating is expected to be lower compared to the sub-zero temperatures. If the cut-off current in CV phase is too small and plating is less, the reverse reaction of the plated lithium may have already completed before the end of the $\mathrm{CV}$ phase. This may increase the detection of plating difficult with the post-charge voltage profiles. Petzl et al. have also mentioned such an issue in their plating studies[19]. They have shown that total plating values went up to a certain SOC level and then came down particularly near to $100 \%$ SOCs. According to them, reversible plating in the $\mathrm{CV}$ phase is the cause for the drop in reversible plating estimation. For plating detection improvement, it is important to stop the charging before the commencement of plating reverse reactions.

In this paper, we present an experiment-based approach to identify the charge cut-off current using the non-destructive voltage relaxation analysis method. Stopping the charge at such a current avoids the reversing in the charging itself and gives scope to detect the maximum lithium plating in the post-charge conditions. The outcome of this work can improve the plating detection and may help in devising a novel plating control strategy.

Further, an attempt to understand the interaction between lithium plating and ageing is made. The study by Ansean et al. concluded that plating is completely reversible and does not cause capacity loss as long as the negative electrode capacity is higher than the positive electrode capacity [5]. As the Loss of Active Material (LAM) occurs at the electrode, negative electrode loses capacity. Once, the electrode capacity drops below the positive electrode's capacity level, part of the lithium plating becomes irreversible. Their simulation models show that the LAM is the cause for the ageing associated with the plating. On the other hand, Yang et al. have attributed the onset of lithium plating to the drop of negative electrode porosity coupled with SEI growth [3]. There is some ambiguity about the reasons for the ageing induced plating because these conclusions are based on the models they developed. In this study, SEI growth impact on the plating is analysed with the help of Electrochemical Impedance Spectroscopy (EIS) plots and VRFs. Finding the causes of the ageing induced plating may allow modifying the plating control according to the ageing mechanism.

\section{Experimental Work}

Experimental work is undertaken to study the plating detection sensitivity to the CV phase cut-off current. In addition, the interaction between the ageing and plating is studied to understand the previous ageing influence on plating and vice versa. Cell selection, experimental setup and the test plan are discussed in this section.

\subsection{Cell details}

Commercial 3.1 Ah lithium nickel cobalt aluminium oxide or NCA-based 18650 battery is used to study lithium plating. Graphite is the negative electrode in this battery. A minimum of three cells for each test scenario is required to ensure statistically meaningful results. Three new cells are used for plating detection and plating impact studies. In addition, aged cells are required to understand how the previous ageing is affecting the plating. Here, ageing is referred in terms of the state of health(SOH) which is the ratio of current capacity retention level to the new cell capacity. Due to limited resources in terms of time and facilities, generating cells with different ageing conditions is not attempted in this work. Instead, thirty-two used cells with unknown previous history are used for an initial screening to select cells with different levels of ageing. Table 1 shows the cell details against the set objectives. 
Table 1. Test objectives and cell requirements

\begin{tabular}{ccc}
\hline Objective & Cells required & Cells used \\
\hline $\begin{array}{c}\text { Improving plating detection } \\
\text { sensitivity }\end{array}$ & fresh cells & 3 cells with $\mathrm{SOH}>98 \%$ \\
$\begin{array}{c}\text { Finding the relationship between } \\
\text { plating and ageing }\end{array}$ & $\begin{array}{c}\text { fresh cells } \\
\text { Aged cells }\end{array}$ & $\begin{array}{l}3 \text { cells with } \mathrm{SOH}>98 \% \\
\text { 2 cells with SOH levels between } 94 \% \text { and } 96 \%\end{array}$ \\
& & 2 cells with levels between $82 \%$ and $88 \%$
\end{tabular}

\subsection{Test plan}

On the selected cells, charge/discharge cycle tests are performed. The overall test plan is presented in Table 2. Cycle tests are categorised broadly into two types: one is the capacity tests and the other one is plating tests. Capacity tests are done at $25^{\circ} \mathrm{C}$ temperature before and after the plating tests. These tests use $\mathrm{C} / 4 \mathrm{CCCV}$ current profile with a cut-off current of $\mathrm{C} / 60$ in the $\mathrm{CV}$ phase for both charge and discharge. Additionally, $\mathrm{C} / 25$ discharge is included at the start and end of the tests to capture the open circuit voltage (OCV) of the cell as part of the capacity tests.

Table 2. Test sequence and conditions

\begin{tabular}{cccc}
\hline Test sequence & Test & Test conditions & temperature \\
\hline 1 & Capacity tests & $0.25 \mathrm{CCCV}$ & $50 \% \mathrm{SOC}$ \\
2 & EIS tests & & $25^{\circ} \mathrm{C}$ \\
3 & Plating test & $1 \mathrm{CCCV}$ & $25^{\circ} \mathrm{C}$ \\
4 & Capacity test & $0.25 \mathrm{CCCV}$ & $25^{\circ} \mathrm{C}$ \\
5 & Plating test & $1 \mathrm{CC}$ with varying CV currents & $25^{\circ} \mathrm{C}$ \\
& & & $05^{\circ} \mathrm{C}$ \\
6 & Capacity tests & $0.25 \mathrm{CCCV}$ & $25^{\circ} \mathrm{C}$ \\
7 & Plating tests & $1 \mathrm{CC}$ with varying CV current & $10^{\circ} \mathrm{C}$ \\
8 & & & \\
& Capacity tests & $0.25 \mathrm{CCCV}$ & $25^{\circ} \mathrm{C}$ \\
9 & Plating tests & $1 \mathrm{CC}$ with varying CV current & $15^{\circ} \mathrm{C}$ \\
10 & Capacity tests & $0.25 \mathrm{CCCV}$ & $25^{\circ} \mathrm{C}$ \\
11 & EIS tests & $50 \% \mathrm{SOC}$ & $25^{\circ} \mathrm{C}$ \\
\hline
\end{tabular}

Plating tests, on the other hand, are performed at different temperatures $\left(5^{\circ} \mathrm{C}, 10^{\circ} \mathrm{C}\right.$ and $\left.15^{\circ} \mathrm{C}\right)$. All the tests use $1 \mathrm{C}$ current $(3 \mathrm{~A})$ in $\mathrm{CC}$ phase but with varying $\mathrm{CV}$ phase cut-off currents. Firstly, At $5^{\circ} \mathrm{C}$, $\mathrm{CV}$ phase impact on the plating detection is studied with cut-off currents ranging from 3 to $0.05 \mathrm{~A}$. Next, the identified optimal cut-off current values for lithium plating detection at $5^{\circ} \mathrm{C}$ temperature is used for the tests under $10^{\circ} \mathrm{C}$ and $15^{\circ} \mathrm{C}$. In addition to the cycle tests, EIS measurements are done at $50 \%$ SOC as part of the reference tests at $25^{\circ} \mathrm{C}$.

\subsection{Test setup}

Fig. 1 shows the experimental setup outline. The tests are run inside a thermal chamber (Model: ESPEC PL-3KPH) to maintain a constant ambient temperature. The cells are connected to a Bitrode cell cycler (Model: MCV 16-100-5) which is controlled by the Bitrode program that runs on the computer. During the operation, the cell temperature generally rises even in the temperature-controlled ambient due to the internal heat generation. Varying CV phase cut-off currents and different levels of ageing together may create large variations in temperature across the cells. This makes the reversible plating amounts comparison difficult since lithium reversing reaction rate depends on temperature [19]. To keep the cell temperature as close as possible to the desired temperature, liquid cooling over the cell surface is used with the support of a LAUDA (Model: Proline RP 845 C) temperature Controller as shown in Figure 2. 


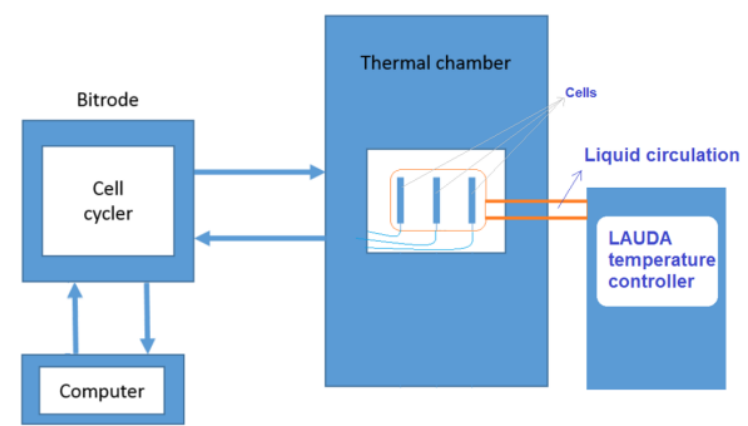

Fig. 1. Experimental setup from the tests

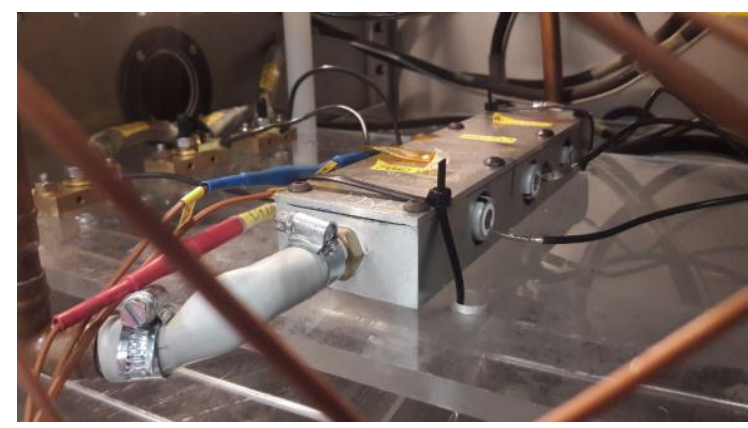

Fig. 2. Test setup with the active cooling mechanism

\section{Results \& Discussions}

\subsection{Detection of lithium plating}

To create the favourable conditions for lithium plating, cells are charged with $1 \mathrm{CCCV}$ at $5^{\circ} \mathrm{C}$. The post-charge cell voltage is monitored for four hours to observe the VRFs for plating detection. Figure $3 \mathrm{a}$ shows the voltage relaxation behaviour of a cell in the post-charge rest at both $25^{\circ} \mathrm{C}$ and $5^{\circ} \mathrm{C}$. The cell voltage is monotonically decreasing with time at room temperature. However, at the low temperature, the cell voltage is recovered in two stages. This additional stage of recovery is due to the reversing reactions of the plated lithium at the graphite electrode [11-13]. By the end of stage 1, the plating reversing completes and cell voltage recovery accelerates once again.

Since the stripping period is in proportion to the amount of reversible plating, the time point at which the second stage starts needs to be identified [13]. This is better captured by performing the rate of change profile, dv/dt, as depicted in Figure 3b. During the lithium strip, the slope of the dv/dt curve reaches a minimum point because of near to flat voltages and attains a maximum point thereafter at the end of reverse reactions. The stripping period $(\tau)$ which is approximately 2030 seconds as shown in the figure indicates that lithium plating reversing reactions lasted over this period.
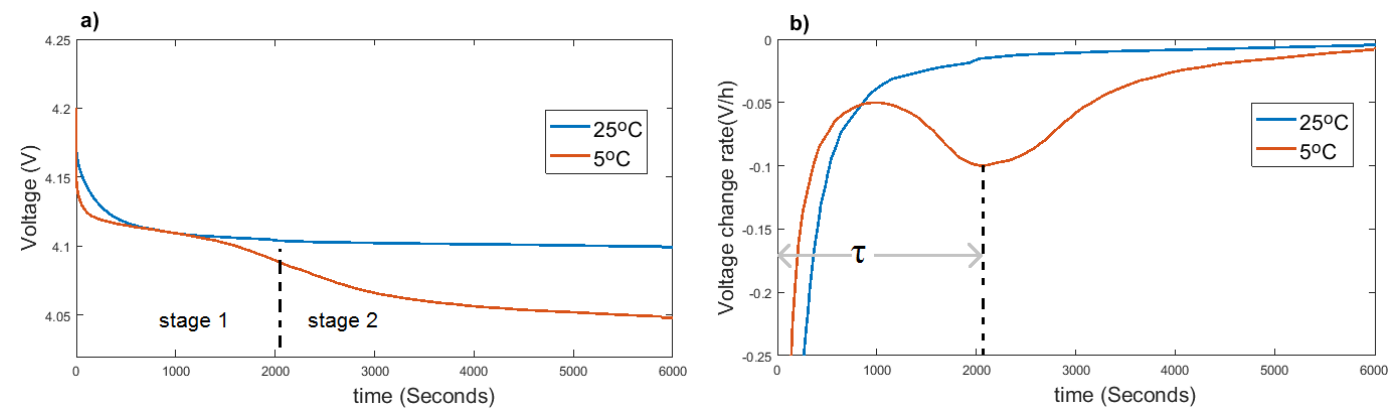

Fig.3. a) Cell voltage relaxation and b) its differential at different temperatures with 1CCCV charging 


\subsection{Improving the detection sensitivity}

As mentioned earlier, lithium plating starts reversing once the electrode potential becomes positive. This may also occur in the CV phase of charging if the cut-off current is small. As demonstrated in Figure 4 , identifying and stopping the charging at the current value where the lithium-plating stops (called $\mathrm{I}_{\text {critical }}$ ) could provide a scope to observe the total reversible plating through VRFs, thus improving the sensitivity of plating detection. To find this critical value of current, cells are tested with different CV cut-off currents as listed in Table 3.

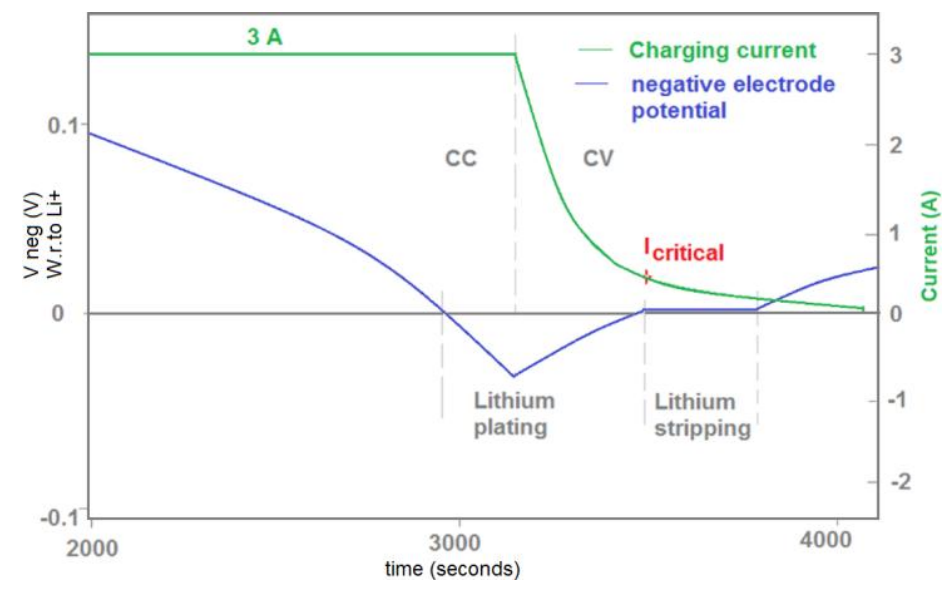

Fig. 4. An illustration of plating and stripping and their link to charge current in CCCV profile[2, 19]

Table 3. Test cases to study the $\mathrm{CV}$ phase cut-off current impact on plating detection

\begin{tabular}{ll}
\hline Test case & Test conditions \\
\hline Case 1 & $1 \mathrm{CC}$ charge alone \\
Case 2 & $1 \mathrm{CCCV}$ with $1 \mathrm{~A}$ as CV cut-off current \\
Case 3 & $1 \mathrm{CCCV}$ with $0.75 \mathrm{~A}$ as CV cut-off current \\
Case 4 & $1 \mathrm{CCCV}$ with $0.5 \mathrm{~A}$ as CV cut-off current \\
Case 5 & $1 \mathrm{CCCV}$ with $0.25 \mathrm{~A}$ as CV cut-off current \\
Case 6 & $1 \mathrm{CCCV}$ with $0.05 \mathrm{~A}$ as CV cut-off current \\
\hline
\end{tabular}

All the six test cases in Table 3 are then performed in sequence on the same cell. In each case, the cell is charged from the fully discharged state to the specified levels and then the cell is rested for four hours. After this, the cells are fully discharged to $2.5 \mathrm{~V}$ with $\mathrm{C} / 3 \mathrm{CCCV}$ and then the next test case starts. The cell voltages in the post-charge relaxation and their time differentials are shown in Figure 5. Since stripping time $(\tau)$ is proportional to the amount of reversible lithium [13], it can be concluded that maximum plating is detected in case 3 . Increasing the cut-off current from 0.75 A reduces the plated lithium thereby decreases the detectable lithium reversion. On the other hand, a decrease in the cut-off value from $0.75 \mathrm{~A}$ does not influence the plated lithium but reduce the detectable lithium. Therefore, the 0.75 cut-off current gives the maximum amount of reversible lithium for plating detection. For a more precise critical current, the current step size in the test cases can be reduced further. 
a)

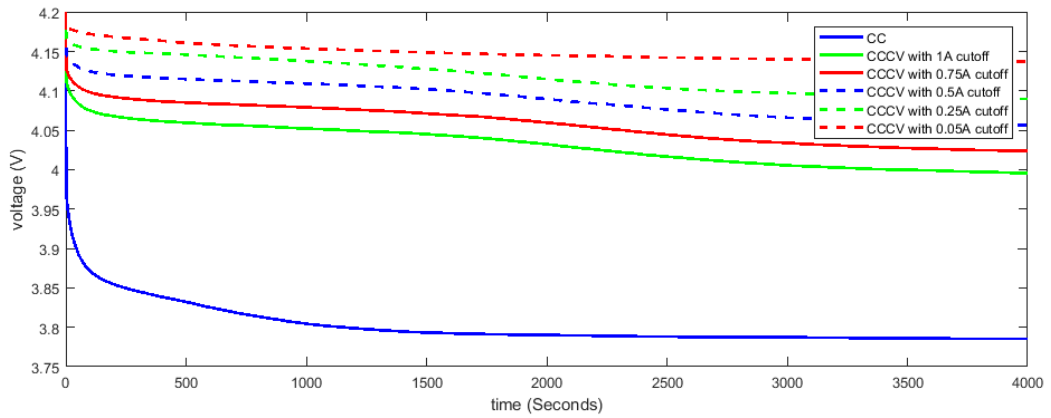

b)

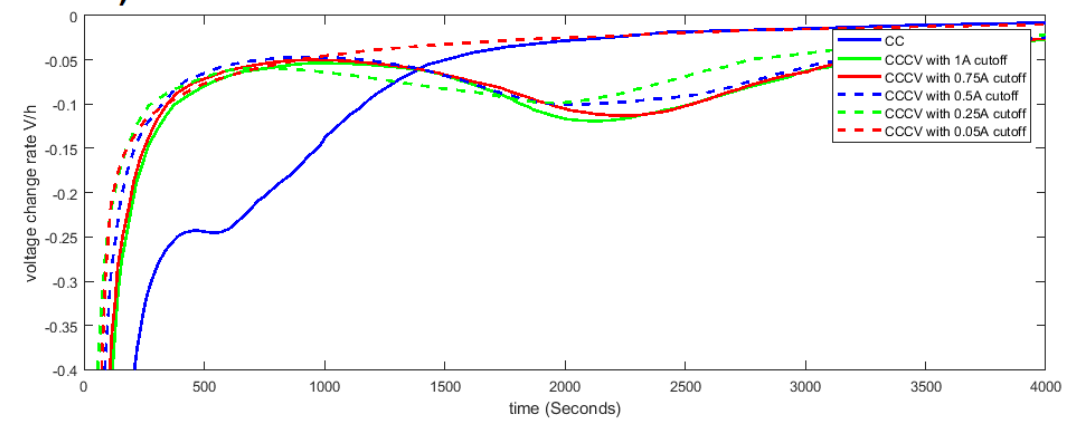

Fig. 5. a) Voltages in rest and b) differential of them with different CV cut-off currents

Table 4. Stripping periods with different cut-off currents

\begin{tabular}{cccccccc}
\hline Cell number & $\tau_{\mathrm{cc}}$ & $\tau_{1 \mathrm{~A}}$ & $\tau_{0.75 \mathrm{~A}}$ & $\tau_{0.5 \mathrm{~A}}$ & $\tau_{0.25 \mathrm{~A}}$ & $\tau_{0.05 \mathrm{~A}}$ & Ratio $\left(\tau_{\mathrm{cc}} / \tau_{\max }\right)$ \\
\hline 4 & 541 & 2114 & 2253 & 2170 & 1854 & 0 & 0.24 \\
5 & 0 & 655 & 519 & 0 & 0 & 0 & 0 \\
6 & 511 & 1823 & 1990 & 2105 & 1760 & 0 & 0.242 \\
7 & 0 & 470 & 504 & 0 & 0 & 0 & 0 \\
8 & 0 & 1351 & 1428 & 1353 & 1020 & 0 & 0 \\
9 & 0 & 1083 & 1205 & 1247 & 910 & 0 & 0 \\
\hline
\end{tabular}

In addition to the plating sensitivity to the cut-off currents, the proportion of the $\mathrm{CC}$ phase contribution to the total plating amount is compared. As shown in Table 4, the stripping time with the CC phase $\left(\tau_{\mathrm{cc}}\right)$ alone is found to be lower than $25 \%$ of the maximum value $\left(\tau_{\max }\right)$ observed for all the cells. This shows that a larger portion of the lithium plating is occurring in the CV phase of charging. This emphasizes that plating control needs a considerable focus on the CV phase to avoid the plating and to reduce the charging time.

\subsection{Plating detection at room temperature}

Apart from the sensitivity study, some interesting findings from the experiment are also reported, which may be helpful for plating studies at room temperature. The plating experiments are continued at different temperatures $\left(5^{\circ} \mathrm{C}\right.$ rise of each step) to check whether the plating is still detectable by VRF at higher temperatures. Since the critical current at which the plating stops is found to be around 0.75A, the plating tests at $10{ }^{\circ} \mathrm{C}$ and $15^{\circ} \mathrm{C}$ are done with a $\mathrm{CV}$ phase cut-off current of $0.75 \mathrm{~A}$. Compared to the results at $5^{\circ} \mathrm{C}$, the tests at $10^{\circ} \mathrm{C}$ show much-reduced stripping periods as depicted in Figure 6 . Increased temperatures reduce the plating and may also increase the reversible plating rate $[6,13]$, thus making the plating detection more difficult. Further, no plating is detected at $15^{\circ} \mathrm{C}$ tests using the VRFs. 


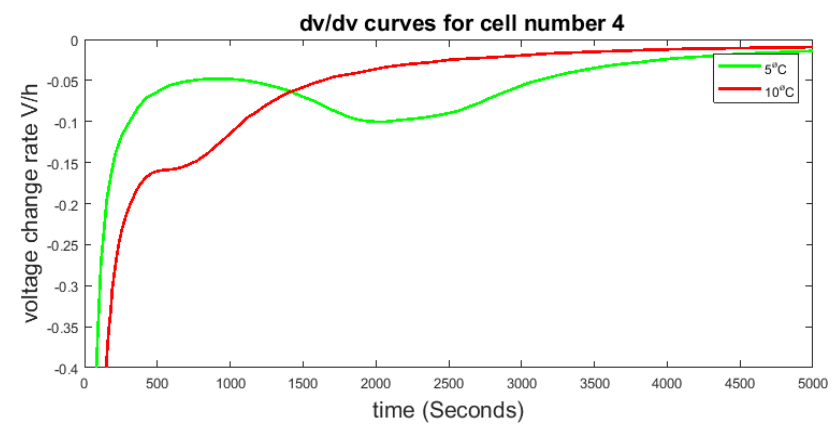

Fig. 6. Comparison of stripping periods at 5 and $10^{\circ} \mathrm{C}$ on a cell while applying the same cut-off current of $0.75 \mathrm{~A}$

However, the charge current profile in the CV phase is found to be different for a subset of the cells. As shown in Figure 7a, cell 4 has a distinct profile compared to cell 3 at room temperature. The current drop of cell 4 is faster from the beginning and much slower in the middle period while that of cell 3 is quite monotonic. In the $\mathrm{CV}$ phase, the cell voltage is maintained at $4.2 \mathrm{~V}$. During the plating reverse, the negative electrode potential is near constant as explained in the previous section. This might lead to a reduced change in the current like cell 4 . To confirm the correlation between the plating and the observed behaviour, current profiles and VRFs captured during the $5^{\circ} \mathrm{C}$ tests are compared. Figure 8 shows the CV phase currents for all the cells at $5^{\circ} \mathrm{C}$. The top five cells with large reversible plating levels have shown the similar behaviour that was observed at $25^{\circ} \mathrm{C}$ confirming its possible link to plating.
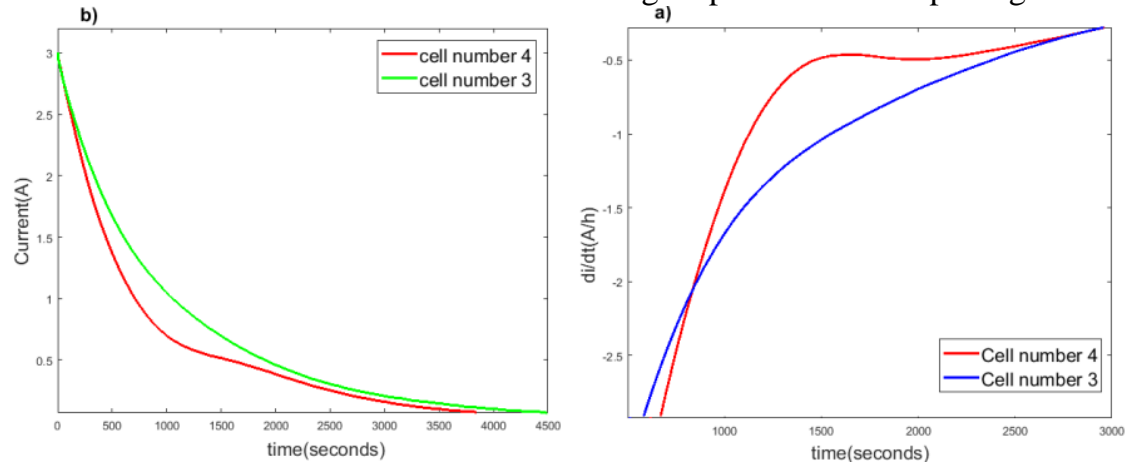

Fig. 7. a) $\mathrm{CV}$ phase current drop and b) its differential at $25^{\circ} \mathrm{C}$ for cells numbered three and four

It appears that the plating detection is possible at higher temperatures by tracking the CV phase current. At the end of lithium stripping, the rate of change in the current rises once again and the corresponding time point can be captured by performing the differential on the CV current as shown in Figure $7 \mathrm{~b}$. However, a detailed study is required to make sure that the observed current profiles are indeed coming from the plating and to find ways to quantify the plating.
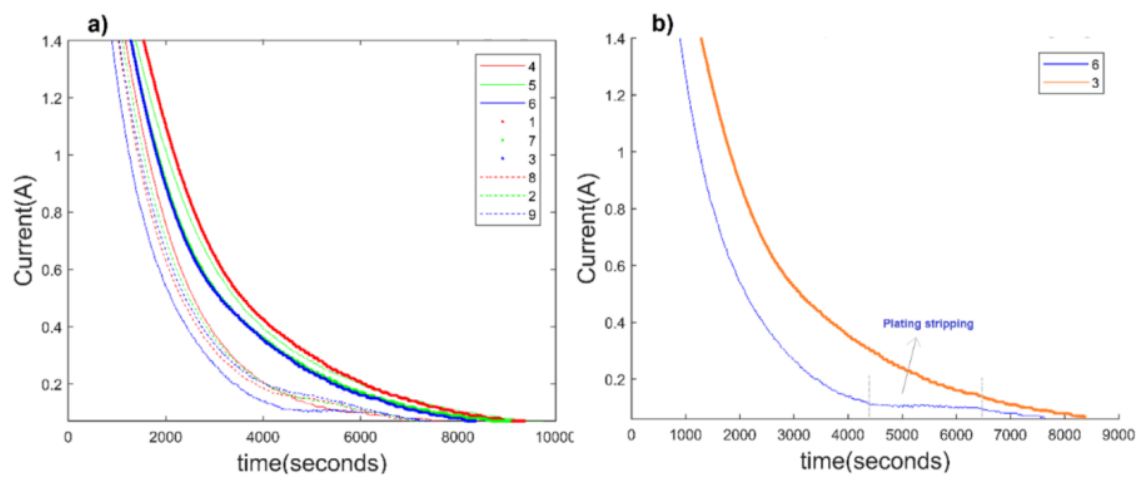

Fig. 8. $\mathrm{CV}$ phase current drop at $5^{\circ} \mathrm{C}$ a) for all the nine cells and b) for cells numbered three and six 


\subsection{Previous ageing influence on plating}

Since previous ageing history is unknown for the aged cells, current capacity levels and EIS plots are used to understand the ageing impact on the plating. Figure 9 shows the EIS plots for all the nine cells before the plating tests along with their $\mathrm{SOH}$ levels measured at the start of the tests. The top five cells, which have the highest capacity retention levels prior to plating tests, showed a single semi-circle in the impedance plot as shown Figure 9a. Figure 9b shows the impedance plots with an additional semi-circle for the other four cells with less retention capacity levels.
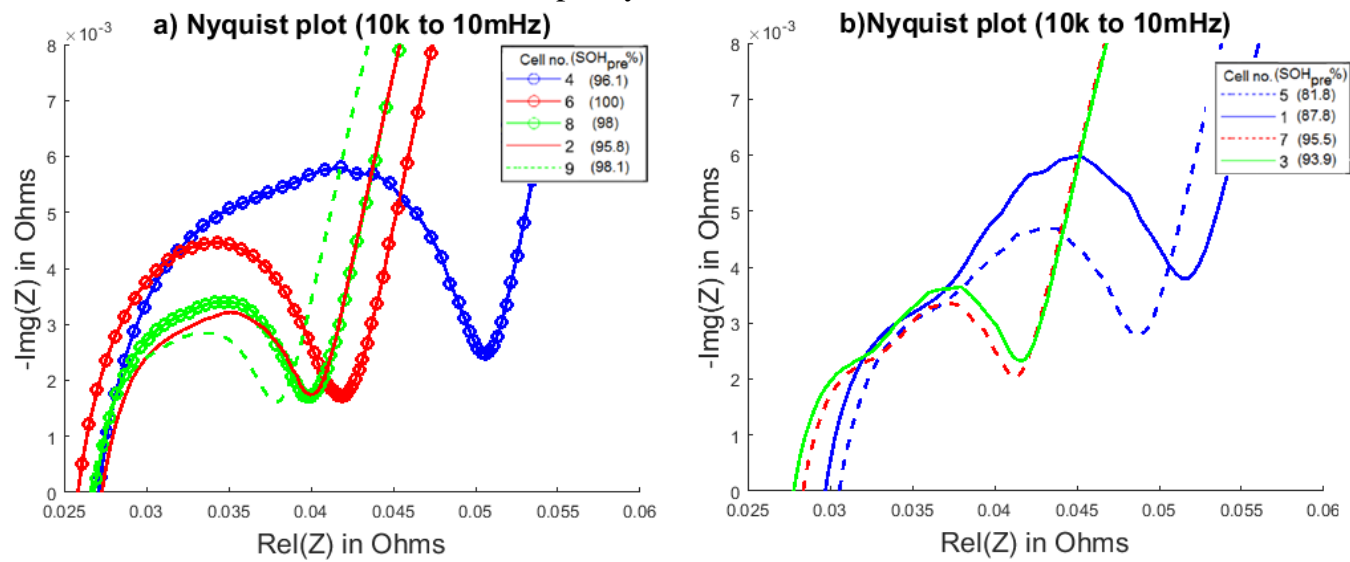

Fig. 9. Impedance spectra of cells with a) single semi-circle and b) with two semi-circles

Generally, cells with higher ageing levels (and less retention capacity) contain a thicker SEI layer compare to the fresh cells. The second semi-circle exhibited by the aged cells might have come from the SEI layer growth. This is confirmed by the EIS plots taken on a separate set of calendar-aged cells stored previously for more than 2 years. Since SEI growth is the dominant ageing that occurs to a cell in storage and the growth of second semi-circle is observed for the calendar-aged cells. It can be reasonably assumed that the second semicircle evolution is due to the SEI layer growth. With the measured EIS plots, SEI layer influence on the plating is analysed.

The five cells with large stripping periods or plating levels in Table 4 have the dominant first semicircle and almost without a second semi-circle, as shown in Figure 9a. Also, these cells are the top five cells in terms of the capacity retention levels (> 95\%) prior to the plating tests. This indicates that these cells are having a thin layer of SEI. On the other hand, the cells with the dominant second semi-circle are the ones that have shown the lower levels of plating. The cell number 1 which lost circa $13 \%$ capacity prior to the plating tests has not shown any signs of plating both in terms of capacity drop and reversible lithium plating observations. Also, this cell has the largest second semi-circle indicating that SEI layer thickness is larger than that of the other cells. Thus, we can conclude that cells with higher levels of SEI layer are less plated compared to the fresh cells.

With the growth of SEI layer or the loss of lithium (LLI), the negative electrode lithiation level at the full charge comes down, and this lower lithiation level leads to a higher voltage at the negative electrode. This may not allow the electrode getting into the negative potential because of the operation at the higher potential.

The electrochemical model based plating studies presented by Yang [3] showed that SEI layer growth is the reason for the plating because of the porosity reduction at the electrode. However, in our tests on the selected cells, the observation is not supporting this idea. Since the construction and initial porosity levels are different from cell to cell, SEI layer growth may not lead to plating in all the cell types.

\subsection{Plating effect on ageing}

All the cells are tested for capacity at the commencement and at the end of plating tests. The results indicate that cell capacity drop is linked to the plating tendency which is represented by the stripping period as shown in Figure 10. Cells 1 and 3 have not displayed the plating behaviour through the VRFs, 
which indicates that reversible plating is zero or lower than the detectable level. In addition, these cells have managed to run more than 40 cycles with less than $1 \%$ capacity fade. This shows that the irreversible plating is also negligible. Thus, it can reasonably be concluded that cell 1 and 3 undergo negligible plating. Cell 7 did show reversible plating but at the lowest level compared to the others that have shown the reversible plating tendency. This cell lost about $1.02 \%$ capacity over the tests, which is the lowest amount compared to the cells showing plating and, is greater than the cells did not undergo plating. While cells numbered 2, 4, 6, 8 and 9 have shown large levels of the reversible plating and they lost capacity considerably $(>2.8 \%)$. It points out that the cells that have shown reversible plating largely have also shown irreversible plating considerably. Cell 5, the most aged cell at $82 \%$ capacity retention levels prior to the tests, has shown lower reversible plating levels but lost huge capacity $(10.22 \%)$. The majority of fade has occurred with this cell at $5^{\circ} \mathrm{C}$ tests. This indicates that the plating is largely irreversible under its aged conditions.

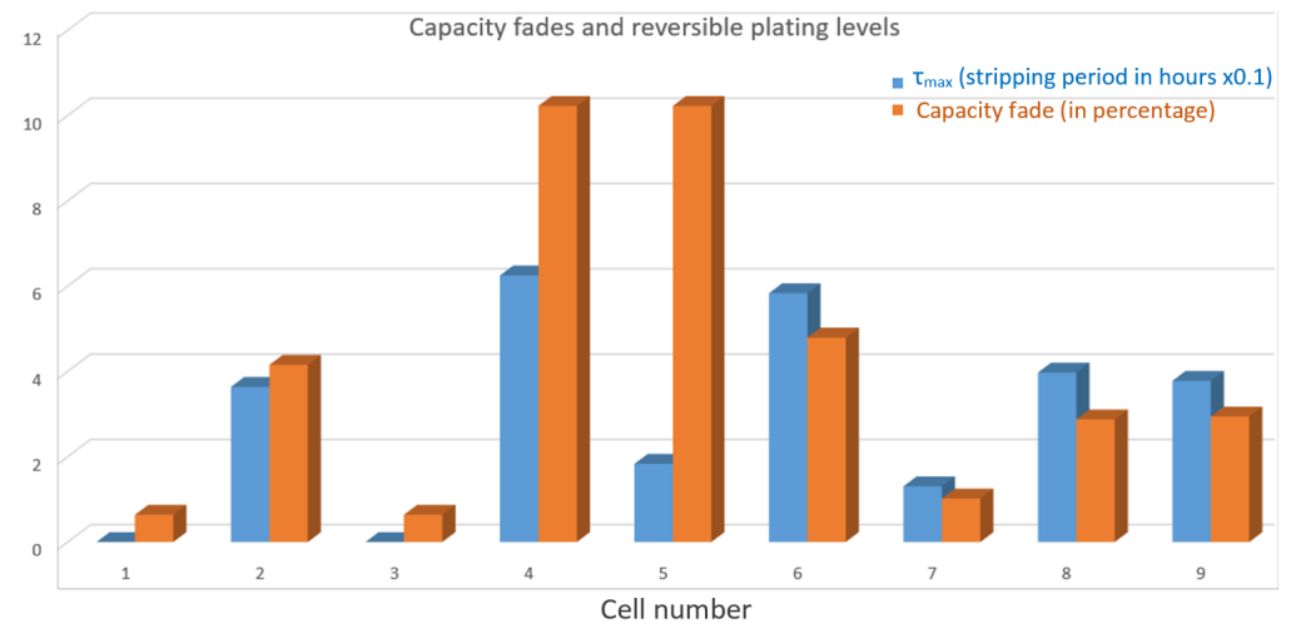

Fig. 10. Plating levels indicated by the maximum stripping period and capacity fade for all the tested cells

To sum up, the cells, which have undergone plating, have lost capacity considerably irrespective of their ageing condition. Ansean et al. [5] have estimated that the plating is completely reversible for the unaged cells and the irreversible portion starts going up with the ageing due to the loss of negative electrode active material. According to the authors, the large capacity of the negative electrode compared to the positive electrode allows the absorption of plated lithium in the post-charge conditions [21]. As the LAM increases, negative electrode material capacity reduces and when it becomes smaller than the positive electrode capacity, the plating starts becoming irreversible. Based on this principle, a fresh cell when it undergoes plating should not face a considerable level of capacity drop. However, our results show that the portion of the plating is irreversible irrespective of the ageing condition. For example, all the fresh cells lost capacity considerably when they underwent plating.

\subsection{Negative feedback between plating and plating aided capacity fade}

With the rise of cycle number under the plated conditions, the stripping time accordingly the plating is reduced. Within first four cycles of the plating tests at $5^{\circ} \mathrm{C}$, there is a reduction of $20 \%$ in stripping time as shown in Figure 11. This could be due to the loss of lithium (LLI) leading to a further plating reduction. As LLI occurs, the lithiation levels of the negative electrode under the full charge starts coming down if the loss of active material at the negative electrode $\left(\mathrm{LAM}_{\mathrm{NE}}\right)$ is slower than the LLI [22]. Ansean et al. [3, 5] pointed out that both the irreversible and reversible plating are going up with the cycle number. This may be true if the plating is leading to porosity reduction at the electrode, consequently increasing the electrode concentration gradients. The increased gradients raise the potential drops at the electrode and thereby causing more plating. Our results, on the other hand, show the negative feedback relationship between the lithium plating and the capacity fade. 

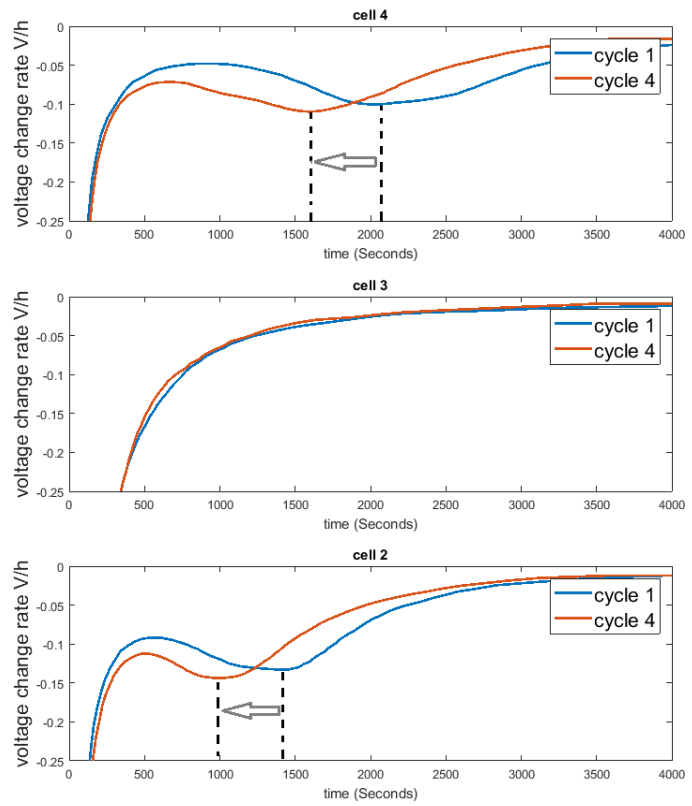

Fig. 11. Stripping time reduction with the increasing cycle number under $5^{\circ} \mathrm{C}$ plating tests

\section{Conclusions and Future Work}

This work analysed the scope for improving the sensitivity of lithium plating detection using the VRF method. The results from the experimental work presented indicate that CV phase cut-off current influences the reversible plating detection. Reversible plating can start stripping in the CV phase if the $\mathrm{CV}$ phase is too long or the cut-off current is too small. Ending the charge before the start of plating reverse improves the plating detection. The optimal cut-off current in the CV phase is identified experimentally to maximize the plating detection sensitivity.

In addition, an attempt to understand the interaction between ageing and plating is made. First, the impact of the previous ageing on the plating is analysed. Cells with large SEI layer are less prone to plating since at temperatures below $10^{\circ} \mathrm{C}$ plating is observed in all the fresh cells but not in the aged cells with thicker SEI layer. Loss of cyclable lithium to the SEI growth is reducing the chances of electrode potentials getting below the Li reference potential. This work used only the EIS plots and capacity retention levels as a qualitative mean to investigate path dependence of lithium-ion cell ageing. Second, plating impact on the capacity fade is discussed. Plated cells irrespective of their ageing conditions have lost capacity considerably in less than 10 cycles at low temperatures. Unlike the previous study[5], our results indicate that plating is not completely reversible for fresh cells since they lost capacity noticeably. Also, higher degradation caused by the plating suggests that plating control strategies need to focus on the pre-emptive measures rather than trying to reduce the plating after its detection. Finally, there is a negative feedback loop between lithium plating and plating induced ageing. The plating tendency is decreasing with the increasing cycle number. The reason may be that the large LLI due to plating is reducing the negative electrode maximum lithiation levels under full charge conditions. Further studies to quantify the ageing in terms of LAM and LLI would be very helpful to clarify the relationship between ageing and plating.

The presented approach to improve the plating detection sensitivity can be utilized in other reversible lithium plating detection methods, such as tomography, discharge DV curves. The experimental results also revealed a promising method of analysing the CV phase charging current for plating detection under room temperature where the conventional VRF method lost its effectiveness. This will be further explored in future research. 


\section{References}

[1] Gao Y. et al., Lithium-ion battery aging mechanisms and life model under different charging stresses. Journal of Power Sources, 2017. 356: 103-114.

[2] Waldmann T, Kasper M, Wohlfahrt-Mehrens M. Optimization of charging strategy by prevention of lithium deposition on anodes in high-energy lithium-ion batteries - electrochemical experiments. Electrochimica Acta, 2015. 178: 525-532.

[3] Yang, XG, et al., Modeling of lithium plating induced aging of lithium-ion batteries: Transition from linear to nonlinear aging. Journal of Power Sources, 2017. 360: p. 28-40.

[4] Legrand N, et al., Physical characterization of the charging process of a Li-ion battery and prediction of Li plating by electrochemical modelling. Journal of Power Sources, 2014. 245: p. 208-216.

[5] Anseán D, et al., Operando lithium plating quantification and early detection of a commercial LiFePO 4 cell cycled under dynamic driving schedule. Journal of Power Sources, 2017. 356: p. 36-46.

[6] Waldmann T, et al., Interplay of Operational Parameters on Lithium Deposition in Lithium-Ion Cells: Systematic Measurements with Reconstructed 3-Electrode Pouch Full Cells. Journal of The Electrochemical Society, 2016. 163(7): p. A1232-A1238.

[7] Uhlmann C, et al., In situ detection of lithium metal plating on graphite in experimental cells. Journal of Power Sources, 2015. 279: p. 428-438.

[8] Bitzer B, Gruhle A. A new method for detecting lithium plating by measuring the cell thickness. Journal of Power Sources, 2014. 262: p. 297-302.

[9] Vetter J, et al., Ageing mechanisms in lithium-ion batteries. Journal of Power Sources, 2005; 147(1-2): 269-281.

[10] Smart, B.V.R.a.M.C. Lithium Plating Behavior in Lithium-ion Cells. ECS Transactions, 2010;25 (36): 241-252.

[11] Zinth V. et al., Lithium plating in lithium-ion batteries at sub-ambient temperatures investigated by in situ neutron diffraction. Journal of Power Sources, 2014; 271: 152-159.

[12] Schindler S, et al., Voltage relaxation and impedance spectroscopy as in-operando methods for the detection of lithium plating on graphitic anodes in commercial lithium-ion cells. Journal of Power Sources, 2016; 304: 170-180.

[13] Von Lüders, C., et al., Lithium plating in lithium-ion batteries investigated by voltage relaxation and in situ neutron diffraction. Journal of Power Sources, 2017; 342:17-23.

[14] Schuster SF., et al., Nonlinear aging characteristics of lithium-ion cells under different operational conditions. Journal of Energy Storage, 2015. 1: p. 44-53.

[15] Bach TC., et al., Nonlinear aging of cylindrical lithium-ion cells linked to heterogeneous compression. Journal of Energy Storage, 2016; 5: p. 212-223.

[16] Burns JC., Stevens DA, Dahn JR. In-Situ Detection of Lithium Plating Using High Precision Coulometry. Journal of the Electrochemical Society, 2015; 162(6): p. A959-A964.

[17] Downie LE. et al., In Situ Detection of Lithium Plating on Graphite Electrodes by Electrochemical Calorimetry. Journal of the Electrochemical Society, 2013; 160(4): p. A588-A594.

[18] Smith AJ., et al., Precision measurements of the coulombic efficiency of lithium-ion batteries and of electrode materials for lithium-ion batteries. Journal of The Electrochemical Society, 2010. 157(2): p. A196.

[19] Petzl M, Danzer MA, Nondestructive detection, characterization, and quantification of lithium plating in commercial lithiumion batteries. Journal of Power Sources, 2014. 254: p. 80-87.

[20] Smart MC, Ratnakumar BV, Effects of electrolyte composition on lithium plating in lithium-ion cells, Journal of The Electrochemical Society, 2011. 158(4): p. A379.

[21] Bennett W. Considerations for Estimating Electrode Performance in Li-Ion Cells. IEEE EnergyTech 2012, 2012: p. 1-5.

[22] Birkl CR, et al., Degradation diagnostics for lithium ion cells. Journal of Power Sources, 2017. 341: p. 373-386. 